nomenclature of course and outcome in bipolar disorders. Bipolar Disord 2009; 11: 453-73.

2 Tohen M, Waternaux CM, Tsuang MT. Outcome in mania. A 4-year prospective follow-up of 75 patients utilizing survival analysis. Arch Gen Psychiatry 1990; 47: 1106-11

3 Tohen M, Zarate CA, Hennen J, Khalsa HM, Strakowski SM, Gebre-Medhin P, et al. The McLean-Harvard First-Episode Mania Study: prediction of recovery and first recurrence. Am J Psychiatry 2003; 160: 2099-107.

Mauricio Tohen, Department of Psychiatry, University of New Mexico Health Science Center, Albuquerque, New Mexico, USA. Email: tohen@uthscsa.edu

doi: $10.1192 /$ bjp.202.2.153a

\section{Is this a non-inferiority trial?}

Crawford et al ${ }^{1}$ have set out to investigate whether screening for suicidal ideation among people who attend primary care services and have signs of depression increases the short-term incidence of feeling that life is not worth living. It seems to me that this is a non-inferiority trial, i.e. the authors want to show that screening is no worse than not screening. This raises a number of design issues.

First, the trial is powered to detect an increase in the proportion who felt their life was not worth living from $30 \%$ to $45 \%$. This seems a clinically large increase and suggests that anything short of a $50 \%$ increase in relative risk is acceptable. Second, having calculated the sample size based on relative risk, they analyse the main results using odds ratios rather than relative risk, so that it is difficult to see what sort of increase in relative risk was found and impossible to see the confidence interval around the relative risk. Third, if this is seen as a non-inferiority trial, arguably screening would be regarded as non-inferior provided that the possibility of the suicidal ideation rate being $50 \%$ worse than non-screening could be ruled out (in the sense that the $95 \%$ confidence interval for the difference in ideation rates would not include $50 \%$ inferiority relative to the non-screened group). ${ }^{2}$ We only have the confidence interval around the odds ratio to go on, but given how wide that is, it is highly likely that the confidence interval would include the $50 \%$ increase in relative risk. For these reasons I think the results should be treated with caution.

1 Crawford MJ, Thana L, Methuen C, Ghosh P, Stanley SV, Ross J, et al. Impact of screening for risk of suicide: randomised controlled trial. $\mathrm{Br} J$ Psychiatry 2011; 198: 379-84.

2 Pocock SJ. The pros and cons of noninferiority trials. Fund Clin Pharmacol 2003; 17: 483-90.

\section{Peter O'Halloran, Lecturer, Queen's University Belfast, UK. Email:}

p.ohalloran@qub.ac.uk

doi: 10.1192/bjp.202.2.154

Authors' reply: Peter O'Halloran raises a good point about this study: it was underpowered. Even slightly higher levels of suicidal thinking among people who are screened for suicide risk would be clinically important and our study was only powered to detect a fairly large difference.

However, at the start of the study we faced a dilemma; a sizeable minority of local general practitioners told us that they were uncomfortable asking people with depression about suicidal thoughts because they were concerned that this could 'make them feel suicidal. ${ }^{1}$ Subsequent discussions with medical students and trainee psychiatrists (and indeed members of the ethics committee that reviewed the study protocol) revealed that these concerns were shared by others. A sample size calculation based on a non-inferiority hypothesis and using a smaller but still clinically important difference in levels of suicidal thinking would have required a sample size several times larger than the one we recruited. As Norman and colleagues have recently pointed out, ${ }^{2}$ sample size calculations are usually a compromise between statistical considerations, economics and logistical constraints. When we embarked on the study we knew that we did not have the resources to recruit a sample large enough to detect a small difference in levels of suicidal thinking among those who were and were not screened. However, we hoped that we could rule out the possibility of a large difference and this is therefore what we set out to do. The 95\% confidence intervals around the odds ratio for the likelihood of suicidal thoughts among those that were screened were broad (0.66-1.18) and are compatible with either higher or lower levels of suicidal thinking in those who are screened compared with those who are not. Despite this limitation, these are the first data that test the veracity of a belief that was held by many and may have been an obstacle to screening for risk of self-harm in this high-risk group.

As we pointed out in the Discussion of our paper, it was not possible to collect data needed to calculate relative risks associated with screening because this would have meant collecting baseline levels of suicidal ideation from all those in the study. This would have exposed those in the control arm of the study to the very factor that the study was designed to examine.

1 Bajaj P, Borreani E, Ghosh P, Methuen C, Patel M, Crawford MJ. Screening for suicidal thoughts in primary care: the views of patients and general practitioners. Ment Health Fam Med 2008; 4: 229-35.

2 Norman G, Monterio S, Salama S. Sample size calculations: should the emperor's clothes be off the peg or made to measure? BMJ 2012; 345: e5278.

Mike J. Crawford, Centre for Mental Health, Faculty of Medicine, Imperial College London, Claybrook Centre, 37 Claybrook Road, London W6 8LN, UK. Email: m.crawford@imperial.ac.uk; Priya Bajaj, Central and North West London NHS Foundation Trust, UK.

doi: 10.1192/bjp.202.2.154a

\section{Delay in starting clozapine and treatment guidelines}

There is a reasonable level of information to suggest that clozapine is effective in patients who have treatment-resistant schizophrenia. Hence, clozapine should be started at the right time so that patients can draw maximum benefit from it. In this vein, the article by Howes et $a l^{1}$ provides important insights into the clinical practice with regard to the use of clozapine. The authors showed that clozapine is delayed by about 4 years and many patients are treated with polypharmacy and receive higher than recommended doses, which is contrary to the recommendations made by several practice guidelines. However, it is important to note that the conclusions drawn about the delay in starting clozapine might not be a true reflection of actual delay, because often patients who are offered clozapine refuse to take it. Hence, some of the delay may be due to lack of agreement of the patient and this in general does not reflect the delay in the clinician offering the medication. It would have been better had the authors extracted the data pertaining to initial offering of clozapine and the number of patients who refused clozapine at the first instance as part of this study. This would have actually given the true clinical picture.

Another issue is the definition of duration of illness used. The authors have defined duration of illness as 'the time from the first recording of the diagnosis of a psychotic illness by a clinician to the present', which may not be a true reflection of duration of 
illness, because there may be varying periods of duration of untreated psychosis and this can have its own treatment implications. Despite these shortcomings, findings of the study suggest that even with a national healthcare system in place and the wider dissemination of treatment guidelines, there is still only a modest impact of these on real clinical practice. The possible effect of treatment guidelines is reflected by the fact that today patients receive fewer trials of other antipsychotics ( $2.8 v .4$ trials $)$ before being started on clozapine compared with earlier studies. ${ }^{2}$

1 Howes OD, Vergunst F, Gee S, McGuire P, Kapur S, Taylor D. Adherence to treatment guidelines in clinical practice: study of antipsychotic treatment prior to clozapine initiation. Br J Psychiatry 2012; 201: 481-5.

2 Taylor DM, Young C, Paton C. Prior antipsychotic prescribing in patients currently receiving clozapine: a case note review. J Clin Psychiatry 2003; 64 $30-4$.

Akhilesh Sharma, Psychiatrist, Department of Psychiatry, Postgraduate Institute of Medical Education \& Research (PGIMER), Chandigarh, India. Email: drakhileshsharma@gmail.com; Sandeep Grover, Assistant Professor, Department of Psychiatry, PGIMER, Chandigarh, India

doi: 10.1192/bjp.202.2.154b

Authors' reply: The first point raised is that the delay to clozapine initiation may not be a true reflection of the actual delay because patients may have been offered clozapine but refused it. This, of course, depends on what delay you are interested in. In our study we used the delay from the point at which treatment guidelines recommend a patient should start clozapine. ${ }^{1}$ In our view this is the key, clinically relevant, delay. However, Sharma \& Grover are right in suggesting that this delay does not necessarily mean that clinicians have delayed offering clozapine, although if this were the case it implies that it has taken on average 4 years for patients to agree to start clozapine. In practice it seems likely that there are a number of patient, clinician and service factors that may underlie the delay we observed in our study. Understanding these will be important if delays are to be reduced in the future. The availability of biomarkers for treatment resistance, as indicated by a recent study, ${ }^{2}$ could also contribute to identifying treatment-resistant patients earlier. Sharma \& Grover also rightly raise the issue that duration of untreated psychosis was not assessed in our study. Consequently, we cannot exclude the possibility that the duration of illness was in fact longer in our sample and thus that the delay to effective treatment was in fact longer.

\section{Declaration of interest}

O.D.H. has been on the speaker bureaux and/or received investigator-initiated charitable research funding from AstraZeneca, Bristol-Myers Squibb, Eli Lilly, and Jansenn-Cilag. D.T. has received consultancy fees, lecturing honoraria and/or research funding from AstraZeneca, Janssen-Cilag, Servier, Sanofi-aventis, Lundbeck, Bristol-Myers Squibb, Novartis, Eli Lilly and Wyeth.

1 Howes OD, Vergunst F, Gee S, McGuire P, Kapur S, Taylor D. Adherence to treatment guidelines in clinical practice: study of antipsychotic treatment prior to clozapine initiation. Br J Psychiatry 2012; 201: 481-5.

2 Demjaha A, Murray RM, McGuire PK, Kapur S, Howes OD. Dopamine synthesis capacity in patients with treatment-resistant schizophrenia. Am J Psychiatry 2012; 169: 1203-10.

Dr Oliver D. Howes, Institute of Psychiatry, De Crespigny Park, London SE5 8AF, UK Email: oliver.howes@kcl.ac.uk; Professor David Taylor, Institute of Psychiatry, London, UK

doi: 10.1192/bjp.202.2.155

\section{Attention-deficit hyperactivity disorder across the lifespan}

Michielsen et al conclude that the personality traits they call attention-deficit hyperactivity disorder (ADHD) 'do not fade or disappear in adulthood. ${ }^{1}$ Yet such a gradual extinction throughout life is precisely what their study proves.

The authors quote prevalences from previous studies as high as $7 \%$ in children and $4.4 \%$ in working-age adults. Their own study shows a prevalence in old age of $2.8 \%$, with higher rates in the 60- to 70 -year age group $(4.0 \%)$ than in those over 70 $(1.1 \%)$. In other words, there is a steady decline in the prevalence of ADHD caseness throughout life, way over and above that which could plausibly be caused by higher mortality among impulsive individuals.

These data show conclusively that, in common with many problematic personality styles, poor attention, impulsivity and hyperactivity tend to gradually lessen in intensity with age. Thus the study is further evidence that ADHD merely represents a cluster of personality traits which, given their high prevalence, cannot even be considered abnormal, rather than a disease entity.

\section{Declaration of interest}

The views expressed are those of the author and are not necessarily shared by his employer.

1 Michielsen $\mathrm{M}$, Semeiin $\mathrm{E}$, Comijs $\mathrm{HC}$, van de Ven $\mathrm{P}$, Beekman ATF, Deeg DJH, et al. Prevalence of attention-deficit hyperactivity disorder in older adults in The Netherlands. Br J Psychiatry 2012; 201: 298-305.

Richard Braithwaite, Consultant Psychiatrist, Isle of Wight NHS Trust, UK. Email: richard.braithwaite@iow.nhs.uk

doi: 10.1192/bjp.202.2.155a

Michielsen et al, ${ }^{1}$ while describing the background and aim of this study, mention that ADHD could lead to significant impairment in older age without providing evidence of such impairment. Certainly from clinical experience and previous studies we know that there are other mental disorders such as depressive illness, anxiety disorder and dementia which are relatively common in older age and likely to cause either similar or more severe impairment. The authors discuss this in some detail in their description of the limitations of this study but fail to consider this when drawing a conclusion about prevalence.

It is essential, according to DSM-IV criteria, for a diagnosis of ADHD to rule out any possibility of the symptoms being better accounted for by another mental disorder. ${ }^{2}$ Unfortunately, the authors do not rule this out while studying the prevalence despite using a diagnostic instrument strongly based on the DSM-IV criteria.

Before we start diagnosing ADHD in older age groups it is important to exclude more prevalent and widely recognised mental health problems such as mild cognitive impairment and dementia. Looking at the diagnostic instrument DIVA 2.0, we can easily identify many symptoms which can be more readily explained by other more prevalent functional and organic illnesses. ${ }^{3}$ This explains why the DIVA 2.0 (as the authors in this study rightly mention) has no evidence for its use in old age. Is retrospective data collected from an older person's recall of being inattentive or hyperactive as a child in different situations valid? More so when DSM-IV clearly advises caution for diagnosing this even in adults without any corroborating information, which was missing in this study.

We would thus suggest extreme caution before we start even suggesting the concept of $\mathrm{ADHD}$ in older adults and taking this 